\title{
Editorial \\ On the way to specifically targeting minimal residual disease?
} Gerhard Gebauer

\author{
Department of Obstetrics and Gynecology, University of Heidelberg, Vossstraße 9, D-69115 Heidelberg, Germany
}

Corresponding author: Gerhard Gebauer, gerhard.gebauer@med.uni-heidelberg.de

Published: 7 October 2008

Breast Cancer Research 2008, 10:112 (doi:10.1186/bcr2148)

This article is online at http://breast-cancer-research.com/content/10/5/112

(c) 2008 BioMed Central Ltd

See related research article by Fehm et al., http://breast-cancer-research.com/content/10/5/R76

\begin{abstract}
The target of all adjuvant systemic therapies after surgery in breast cancer is the eradication of a minimal subclinical residual disease. Although it is well known that tumor cell dissemination takes place already at an early stage of the disease, little is known about the tumorbiological parameters of these residual cells. Selection of patients eligible for adjuvant endocrine therapies is based on the analysis of receptor expression in the primary tumor - although the analysis is directed against disseminated tumor cells, these cells may vary in receptor expression in comparison with the primary tumor.
\end{abstract}

In the current issue of Breast Cancer Research, Fehm and colleagues present an analysis of estrogen receptor expression indicating considerable molecular differences between the primary tumor and the corresponding disseminated tumor cells (DTC) [1].

More than 20 years ago Bernhard Fisher raised the hypothesis that breast cancer is a systemic disease even at an early stage, rather than just locally restricted [2]. This opened the gate to a dramatic change in therapeutic strategies, from radical surgery to new concepts based on less radical local therapy combined with additional therapeutic options such as radiotherapy, endocrine treatment and cytotoxic treatment. These modern multimodal strategies considerably improved the outcome of breast cancer patients over the past decades and proved - due to the success of this approach - the hypothesis of breast cancer as a primarily systemic disease [3].

The majority of early breast cancer patients to date receive a combination of surgery, radiotherapy, endocrine treatment, cytotoxic treatment or immunologic treatment. But do all patients treated by these often highly toxic and side-effectcausing regimens really need and benefit from these treatments? We know that only a minority of approximately $10 \%$ to $20 \%$ of patients do really benefit from systemic therapy [4]. We still do not, however, have suitable para- meters predicting response to therapy or, more importantly, the need for systemic therapy. Since some patients will have long-term benefit from these strategies, the massive overtreatment of the majority of breast cancer patients is currently well accepted in the scientific community.

Since the 1980s DTC have been described in the bone marrow of early breast cancer patients as well as in patients with various other tumors $[5,6]$. The presence of these cells seemed to prove the concept of a metastatic spread of tumor cells already at a very early stage. The follow-up data of these patients published over recent years, however, have clearly indicated that the presence of these cells alone does not necessarily reflect an active metastatic disease in every single patient $[7,8]$. Although DTC are detectable in up to $40 \%$ of early breast cancer patients, the majority remains diseasefree even over 10 years and longer. DTC therefore apparently indicate a high-risk situation but not always an ongoing metastatic tumor cell spread. Nevertheless, the detection of DTC in bone marrow and blood has become one of the most promising parameters for identifying high-risk breast cancer patients - and may, moreover, allow monitoring of patients under therapy in order to determine the therapy response in the future.

Analysis of molecular parameters such as the expression of Her2 receptors, estrogen receptors or progestin receptors became routine clinical practice in breast cancer. These parameters allow selection of patients eligible for specific therapeutic strategies targeting receptor-expressing cells. For example, treatment of breast cancer with tamoxifen is one of the oldest strategies for targeted therapy in estrogen receptor-positive patients. These parameters, however, are routinely analyzed in the primary tumor itself. The receptor expression between primary tumor and metastatic tissue may vary [9]. The considerable number of therapy failures may be explained by these molecular differences between cells of the

DTC $=$ disseminated tumor cells. 
primary tumor - surgically removed and histopathologically analyzed - and the remaining DTC, which may later cause tumor recurrence. If one may accept the concept that tumor cell dissemination takes place already in small tumors and DTC may eventually be detectable in various organs such as bone marrow, it appears consequent to obtain molecular details from those tumor cells that remain behind after surgery - the so-called minimal residual disease. These cells would be the real targets for any systemic therapy in order to prevent them forming a clinically relevant metastatic disease.

Analyzing a large cohort of patients, Fehm and colleagues demonstrated surprisingly dramatic differences in estrogen receptor expression between the primary tumor and the corresponding DTC. Of 107 patients with DTC in the bone marrow, only in 30 cases could identical results for estrogen receptor expression be obtained between the primary tumor and the corresponding DTC [1]. These data became even more complex taking into consideration the heterogeneity of estrogen receptor expression between different DTC of the same patient.

The results of Fehm and colleagues on the estrogen receptor status of DTC force us to rethink our understanding of treatment success and failure based on the old-fashioned parameters obtained from a primary tumor as the predictor for therapy response - a tumor that already is surgically removed at the time we even start thinking of adjuvant therapeutic options for a particular patient. Fehm and colleagues' data may ultimately suggest new and more appropriate parameters to consider systemic therapies in breast cancer - obtainable at various time points in various clinical situations, leading to more individualized treatment options. Nevertheless, any new strategy based on the characterization of DTC will have to be proven by the strongest outcome parameter known in oncology - the improvement of survival.

\section{Competing interests}

The author declares that they have no competing interests.

\section{References}

1. Fehm TN, Krawczyck N, Solomayer E, Becker-Pergola G, DurrStorzer S, Neubauer H, Seeger H, Staebler A, Wallwiener D, Becker S: ER alpha-status of disseminated tumor cells in bone marrow of primary breast cancer patients. Breast Cancer Res 2008, 10:R76.

2. Fisher B: Laboratory and clinical research in breast cancer - a personal adventure: the David A. Karnofsky memorial lecture. Cancer Res 1980, 40:3863-3874.

3. Punglia RS, Morrow M, Winer EP, Harris JR: Local therapy and survival in breast cancer. N Engl J Med 2007, 356:2399-2405.

4. Effects of chemotherapy and hormonal therapy for early breast cancer on recurrence and 15-year survival: an overview of the randomised trials. Lancet 2005, 365:1687-1717.

5. Gebauer G, Fehm T, Merkle E, Beck EP, Lang N, Jager W: Epithelial cells in bone marrow of breast cancer patients at time of primary surgery: clinical outcome during long-term follow-up. J Clin Oncol 2001, 19:3669-3674.

6. Cote RJ, Rosen PP, Lesser ML, Old LJ, Osborne MP: Prediction of early relapse in patients with operable breast cancer by detection of occult bone marrow micrometastases. J Clin Oncol 1991, 9:1749-1756.
7. Braun S, Marth C: Circulating tumor cells in metastatic breast cancer - toward individualized treatment? N Engl J Med 2004, 351:824-826.

8. Braun S, Vogl FD, Naume B, Janni W, Osborne MP, Coombes RC, Schlimok G, Diel IJ, Gerber B, Gebauer G, Pierga JY, Marth C, Oruzio D, Wiedswang G, Solomayer EF, Kundt G, Strobl B, Fehm T, Wong GY, Bliss J, Vincent-Salomon A, Pantel K: A pooled analysis of bone marrow micrometastasis in breast cancer. N Engl J Med 2005, 353:793-802.

9. Andersen J, Poulsen HS: Relationship between estrogen receptor status in the primary tumor and its regional and distant metastases. An immunohistochemical study in human breast cancer. Acta Oncol 1988, 27:761-765. 